\title{
ON THE SCHAUDER FIXED POINT THEOREM
}

\author{
LECH GÓRNIEWICZ \\ Department of Mathematics and Informatics, Nicholas Copernicus University, \\ ul. Chopina 12/18, Toruń, Poland \\ E-mail: gorn@mat.uni.torun.pl \\ DANUTA ROZPEOCH-NOWAKOWSKA \\ Department of Mathematics and Informatics, Nicholas Copernicus University, \\ ul. Chopina 12/18, Toruń, Poland \\ E-mail: nowa@mat.uni.torun.pl
}

\begin{abstract}
The paper contains a survey of various results concerning the Schauder Fixed Point Theorem for metric spaces both in single-valued and multi-valued cases. A number of open problems is formulated.
\end{abstract}

1. Introduction. The famous Schauder Fixed Point Theorem proved in 1930 (see [S]) was formulated as follows:

Satz II. Let $H$ be a convex and closed subset of a Banach space. Then any continuous and compact map $F: H \rightarrow H$ has a fixed point.

This theorem still has an enormous influence on the fixed point theory and on the theory of differential equations. Therefore it seems to be useful to present the most general current results in this area and formulate some problems which are still open.

The paper is organized as follows: in Sections 2 and 3 we present a background concerning the Lefschetz number and ANR-spaces. Then in Section 4 we consider the classes of mappings for which the Schauder Fixed Point Theorem is true.

The most general formulation of the Schauder Fixed Point Theorem is presented in Section 5 where several open problems are also stated.

In the last section we describe a large class of multivalued maps, so-called admissible (see $[\mathrm{LG}]$ or $[\mathrm{FG}]$ ), for which all results of Section 4 remain true.

1991 Mathematics Subject Classification: Primary 55M20; Secondary 54H25, 47H10, 47H04.

Key words and phrases: fixed point, absolute retract, degree, set-valued operators.

The paper is in final form and no version of it will be published elsewhere. 


\section{The Lefschetz number}

2.1. The ordinary trace. In this section all vector spaces are assumed to be linear spaces over the field of rational numbers $\mathbb{Q}$ and all maps between such spaces are linear. We recommend $[\mathrm{AG}]$ for details. For an endomorphism of a finite dimensional vector space E (i.e. linear mapping from $\mathrm{E}$ into itself) $L: E \rightarrow E$, we let $\operatorname{tr} L$ denote the ordinary trace of $L$, i.e. the trace of its matrix representation.

We now recall well-known properties of the trace function tr:

Proposition 2.1.1. Assume that in the category of finite dimensional vector spaces the following diagram commutes

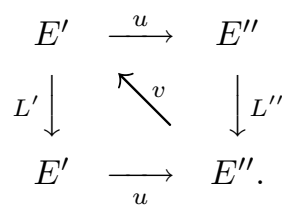

Then $\operatorname{tr} L^{\prime}=\operatorname{tr} L^{\prime \prime}$, or equivalently $\operatorname{tr}(v u)=\operatorname{tr}(u v)$.

Proposition 2.1.2 Given a commutative diagram of finite dimensional vector spaces with exact rows

we have $\operatorname{tr} L=\operatorname{tr} L^{\prime}+\operatorname{tr} L^{\prime \prime}$.

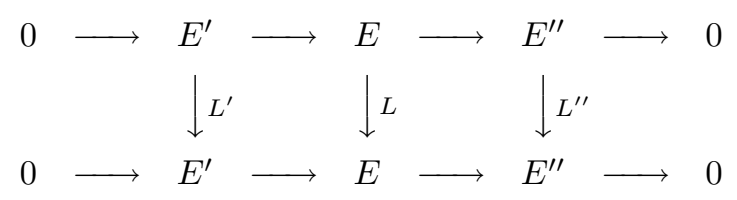

Definition 2.1.3. Let $E=\left\{E_{q}\right\}$ be a graded vector space i.e. a sequence of vector spaces indexed by natural numbers. We say that $E$ is of finite type provided:

(i) $\operatorname{dim} E_{q}<\infty$ for all $q$,

(ii) $E_{q}=0$ for almost all $q$. If $L=\left\{L_{q}\right\}$ is an endomorphism of the space $E$ (i.e. $\left.L_{q}: E_{q} \rightarrow E_{q}\right)$ then the Lefschetz number $\lambda(L)$ of $L$ is defined by

$$
\lambda(L)=\sum_{q}(-1)^{q} \operatorname{tr}\left(L_{q}\right) .
$$

Now we are going to define the generalized trace.

2.2. The Leray trace. Let $L: E \rightarrow E$ be an endomorphism of an arbitrary vector space $E$. Denote by $L^{(n)}: E \rightarrow E$ the $n$-th iterate of $L$ and observe that the kernels

$$
\operatorname{Ker} L \subset \operatorname{Ker} L^{(2)} \subset \ldots \subset \operatorname{Ker} L^{(n)} \subset \ldots
$$

form an increasing sequence of subspaces of $E$.

We put

$$
N(L)=\bigcup_{n \geq 1} \operatorname{Ker} L^{(n)} .
$$

By definition

$$
x \in N(L) \Leftrightarrow L^{(n)}(x)=0
$$

for some $n$. Obviously, $L$ maps $N(L)$ into itself and, therefore, induces the endomorphism $\widetilde{L}: \widetilde{E} \rightarrow \widetilde{E}$, where $\widetilde{E}=E / N(L)$ is the factor space. 
Proposition 2.2.1. $\widetilde{L}: \widetilde{E} \rightarrow \widetilde{E}$ is a monomorphism.

Definition 2.2.2. Let $L: E \rightarrow E$ be an endomorphism of a vector space $E$. We say that $L$ is admissible provided the factor space $\widetilde{E}$ is finite dimensional. For such $L$, we define the (generalized) trace $\operatorname{Tr} L$ of $L$ by putting $\operatorname{Tr} L=\operatorname{tr} \widetilde{L}$.

Below we list important properties of the generalized trace.

Theorem 2.2.3. (1) Let $L: E \rightarrow E$ be an endomorphism. If $\operatorname{dim} E<\infty$, then $\operatorname{Tr} L=\operatorname{tr} L$.

(2) Assume that in the category of arbitrary vector spaces the following diagram commutes

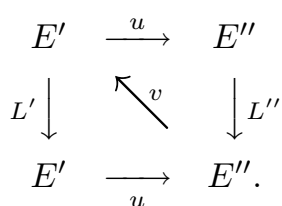

Then if one of the maps $L^{\prime}$ or $L^{\prime \prime}$ is admissible, then so is the other and in that case $\operatorname{Tr} L^{\prime}=\operatorname{Tr} L^{\prime \prime}$.

THEOREM 2.2.4. Given a commutative diagram of vector spaces with exact rows

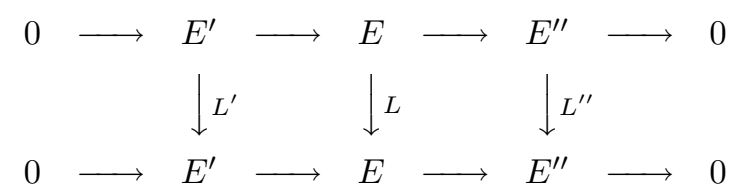

the endomorphism $L$ is admissible if and only if both $L^{\prime \prime}$ and $L^{\prime}$ are admissible and in that case

$$
\operatorname{Tr} L=\operatorname{Tr} L^{\prime \prime}+\operatorname{Tr} L^{\prime} .
$$

Now we are in position to define the generalized Lefschetz number.

2.3. The Leray endomorphisms. Let $L=\left\{L_{q}\right\}$ be an endomorphism of a graded vector space $E=\left\{E_{q}\right\}$ into itself. By $\widetilde{L}=\left\{\widetilde{L}_{q}\right\}$ we denote the induced endomorphism on the graded vector space $\widetilde{E}=\left\{\widetilde{E}_{q}\right\}$.

DEFINITION 2.3.1. We say that $L$ is a Leray endomorphism provided the graded vector space $\widetilde{E}=\left\{\widetilde{E}_{q}\right\}$ is of finite type. For such $L$ we define the (generalized) Lefschetz number $\Lambda(L)$ of $L$ by putting:

$$
\Lambda(L)=\sum_{q}(-1)^{q} \operatorname{Tr}\left(L_{q}\right) .
$$

The following two theorems are immediate consequence of 2.2.3 and 2.2.4, respectively.

Definition 2.3.2. Assume that in the category of graded vector spaces the following diagram commutes

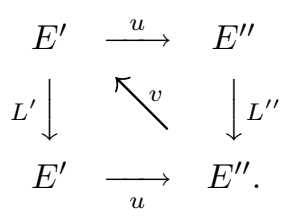


Then if any of the maps $L^{\prime}$ or $L^{\prime \prime}$ is Leray endomorphism, then so is the other and in that case $\Lambda\left(L^{\prime}\right)=\Lambda\left(L^{\prime \prime}\right)$.

TheOREM 2.3.3. Let

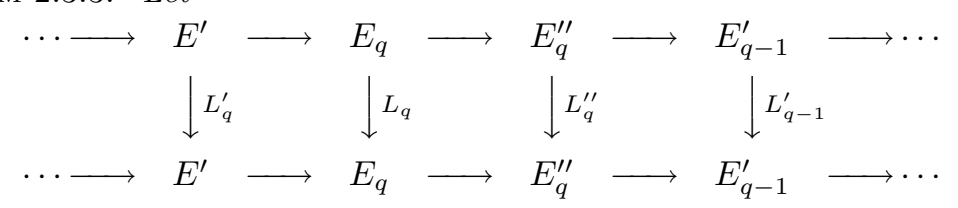

be a commutative diagram of vector spaces in which the rows are exact. If both $L=\left\{L_{q}\right\}$ and $L^{\prime}=\left\{L_{q}^{\prime}\right\}$ are the Leray endomorphisms on $E=\left\{E_{q}\right\}$ and $E^{\prime}=\left\{E_{q}^{\prime}\right\}$ respectively, then so is $L^{\prime \prime}=\left\{L_{q}^{\prime \prime}\right\}$ on $E^{\prime \prime}=\left\{E_{q}^{\prime \prime}\right\}$. Moreover, in that case, we have:

$$
\Lambda(L)=\Lambda\left(L^{\prime}\right)-\Lambda\left(L^{\prime \prime}\right) .
$$

Definition 2.3.4. An endomorphism $L: E \rightarrow E$ is called weakly nilpotent if for every $x \in E$ there exists a natural number $n=n_{x}$ such that:

$$
L^{n}(x)=0 \text {. }
$$

From 2.3.4 it is easy to see that $L: E \rightarrow E$ is weakly nilpotent if and only if $N(L)=E$. Therefore we get:

Proposition 2.3.5. Any weakly nilpotent endomorphism $L: E \rightarrow E$ is admissible and $\operatorname{Tr}(L)=0$.

Assume that $E=\left\{E_{q}\right\}$ is graded vector space and $L=\left\{L_{q}\right\}: E \rightarrow E$ is an endomorphism. We say that $L$ is weakly nilpotent if and only if $L_{q}$ is weakly nilpotent for every $q$.

From 2.3.5 we deduce:

Proposition 2.3.6. Any weakly nilpotent endomorphism $L: E \rightarrow E$ of graded vector space $E$ is a Leray endomorphism and $\Lambda(L)=0$.

3. Lefschetz maps. By a pair of spaces, $(X, A)$, we understand a pair of a metric space $X$ and one of its subsets, $A$. A pair of the form $(X, \emptyset)$ will be identified with the space $X$. By a map, $f:(X, A) \rightarrow(Y, B)$, we understand a continuous (single-valued) map $f: X \rightarrow Y$ satisfying the condition $f(A) \subset B$.

Let $H$ be the Čech homology functor with compact carriers and coefficients in the field of rational numbers $\mathbb{Q}$ (comp. $[\mathrm{LG}]$ ).

For a pair $(X, A)$ let us consider the graded vector space $H(X, A)=\left\{H_{q}(X, A)\right\}$. For a continuous map $f:(X, A) \rightarrow(Y, B), H(f)$ is the induced linear map $f_{*}=\left\{f_{q}\right\}$, where $f_{q}: H_{q}(X, A) \rightarrow H_{q}(Y, B)$.

We shall say that $f:(X, A) \rightarrow(X, A)$, has a fixed point provided that there exists $x_{0} \in X$ such that $f\left(x_{0}\right)=x_{0}$.

Definition 3.1. A continuous mapping $f:(X, A) \rightarrow(X, A)$ is called a Lefschetz map (with respect to $H$ ) provided $f_{*}: H(X, A) \rightarrow H(X, A)$ is Leray endomorphism. For such $f$ we define the Lefschetz number $\Lambda(f)$ of $f$ by putting

$$
\Lambda(f)=\Lambda\left(f_{*}\right) .
$$


From 2.3.2 we get

Proposition 3.2. If the maps $f$ and $g$ are homotopic, then their Lefschetz numbers (if defined) coincide, i.e. $\Lambda(f)=\Lambda(g)$.

THEOREM 3.3. Assume that in the category of pairs of spaces and continuous maps the following diagram commutes

Then

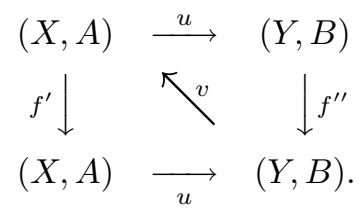

(i) if one of the maps $f^{\prime}$ or $f^{\prime \prime}$ is a Lefschetz map, then so is the other and in that case $\Lambda\left(f^{\prime}\right)=\Lambda\left(f^{\prime \prime}\right)$,

(ii) $f^{\prime}$ has a fixed point if and only if $f^{\prime \prime}$ does.

Given a continuous mapping $f:(X, A) \rightarrow(X, A)$ we denote by $f_{X}: X \rightarrow X$ and $f_{A}: A \rightarrow A$ the evident contractions of $f$.

From 2.3.3 we obtain (see $[\mathrm{CB}]$ )

Theorem 3.4. Let $f:(X, A) \rightarrow(X, A)$ be a mapping. If any two of $f, f_{X}$ and $f_{A}$ are Lefschetz maps, then so is the third map $f$ and in that case

$$
\Lambda(f)=\Lambda\left(f_{X}\right)-\Lambda\left(f_{A}\right) .
$$

Recall that a continuous mapping $f: X \rightarrow X$ is called homologically trivial with respect to the functor $H$ provided the induced homomorphisms $f_{* q}: H_{q} \rightarrow H_{q}$ are trivial for $q \geq 1$ and $f_{* 0}: H_{0}(X) \simeq H_{0}(X)$. A space $X$ is said to be acyclic (with respect to the $H)$ provided

(i) $X$ is non-empty,

(ii) $H_{0}(X) \approx \mathbb{Q}$,

(iii) $H_{q}(X)=0$ for all $q \geq 0$.

The following result is self-evident.

Theorem 3.5. Let $f: X \rightarrow X$ be continuous and assume that any of the following conditions is satisfied:

(1) $f(X)$ is contained in an acyclic subset $A$ of $X$,

(2) $H_{0}(X) \approx \mathbb{Q}$ and $f: X \rightarrow X$ is homologically trivial,

(3) $H_{0}(X) \approx \mathbb{Q}$ and $f: X \rightarrow X$ is homotopic to a constant map.

Then, $f$ is a Lefschetz map and $\Lambda(f)=1$.

3.1. Absolute retracts.

DEFINITION 3.1.1. A space $Y$ is called absolute retract (absolute neighbourhood retract) whenever for any metrizable $X$ and closed $A \subset X$ each $f: A \rightarrow Y$ is extendable over $X$ (over an open neighbourhood $U$ of $A$ in $X$ ).

We use notation: $Y \in \operatorname{AR}(Y \in \mathrm{ANR})$. 
The following theorem characterizes AR (ANR) in terms of retraction property (up to homeomorphism):

THEOREM 3.1.1 ([B]). A metrizable space is an AR (ANR) if and only if it is a retract of (some open subset of) some normed space.

Observe that any normed space is homologically trivial and therefore from 3.1.2 we deduce that any AR-space $\mathrm{X}$ is homologically trivial, too. Hence, in view of 3.5 we get

Propositon 3.1.3. Let $X \in \mathrm{AR}$ and let $f: X \rightarrow X$ be a continuous map. Then $f$ is a Lefschetz map and $\Lambda(f)=1$.

Now as a simple consequence of 3.1.2 we obtain:

Proposition 3.1.4. If $X \in \mathrm{ANR}$ and $U$ is an open subset of $X$, then $U \in \mathrm{ANR}$.

A continuous map $f: X \rightarrow X$ is called compact provided the closure $\overline{f(X)}$ of $f(X)$ in $X$ is a compact set. We shall write: $f \in \mathbb{K}(X)$.

We shall use the following theorem due to A. Granas [AG1]:

TheOREM 3.1.5. Let $X \in \mathrm{ANR}$ and let $f: X \rightarrow X$ be a compact map then:

(1) $f$ is a Lefschetz map,

(2) $\Lambda(f) \neq 0$ implies that $f$ has a fixed point.

We recommend $[\mathrm{B}],[\mathrm{AG}]$, [AG1] for details concerning this section.

4. Compact absorbing contraction maps. Following [FG] (see also [F1], [F2], [F3]) we shall define some classes of maps of a great importance in our work.

Let $X$ be a metric space.

Definition 4.1. A continuous map $f: X \rightarrow Y$ is called locally compact provided that, for each $x \in X$ there exists an open subset $V$ of $X$ such that $x \in V$ and the restriction $\left.f\right|_{V}$ of $f$ to $V$ is compact.

All mappings considered in this section are assumed to be locally compact.

Definition 4.2. A map $f: X \rightarrow X$ is said to be a compact absorbing contraction if there exists an open set $U$ of $X$ such that $\overline{f(U)}$ is a compact of $U$ and $X \subset \bigcup_{i=0}^{\infty} f^{-i}(U)$.

We use a notation: $f \in \mathbb{C A C}(X)$.

Definition 4.3. A map $f: X \rightarrow X$ is called eventually compact if there exists an iterate $f^{n}: X \rightarrow X$ of $f$ such that $f^{n}$ is compact.

We use a notation: $f \in \mathbb{E} \mathbb{C}(X)$.

Definition 4.4. A map $f: X \rightarrow X$ is called compact attraction if there exists a compact $K$ of $X$ such that, for each open neighbourhood $V$ of $K$, we have $X \subset$ $\bigcup_{i=0}^{\infty} f^{-i}(V)$. The compact $K$ is then called an attractor for $f$.

We use a notation: $f \in \mathbb{C} A(X)$. 
Definition 4.5. A map $f: X \rightarrow X$ such that

$$
\bigcup_{n=1}^{\infty}\left\{f^{n}(x)\right\} \text { is relatively compact for every } x \in X
$$

is called asymptotically compact if the set $C_{f}=\bigcap_{i=0}^{\infty} f^{i}(X)$ is a non-empty, relatively compact subset of $X$. The set $C_{f}$ is called the center of $f$.

We use a notation: $f \in \mathbb{A} \mathbb{S} \mathbb{C}(X)$.

Note that any eventually compact map is a compact attraction, i.e. $\mathbb{E} \mathbb{C}(X) \subset \mathbb{C A}(X)$. However, we need the following lemma to prove Proposition 4.7.

Lemma 4.6. $f \in \mathbb{E} \mathbb{C}(X) \Rightarrow f \in \mathbb{C} \mathbb{A} \mathbb{C}(X)$.

Proof. Let $f: X \rightarrow X$ be an eventually compact map such that $K^{\prime}=\overline{f^{n}(X)}$ is compact. Define $K=\bigcup_{i=0}^{n-1} f^{i}\left(K^{\prime}\right)$, we have:

$$
f(K) \subset \bigcup_{i=1}^{n} f^{i}\left(K^{\prime}\right) \subset K \cup f^{n}(X) \subset K \cup K^{\prime} \subset K .
$$

Since $f$ is locally compact, there exists an open neighbourhood $V_{0}$ of $K$ such that $L=$ $\overline{f\left(V_{0}\right)}$ is compact.

There exists a sequence $\left\{V_{1}, \ldots, V_{n}\right\}$ of open subsets of $X$ such that $L \cap \overline{f\left(V_{i}\right)} \subset V_{i-1}$ and $K \cup f^{n-i}(L) \subset V_{i}$ for all $i=1, \ldots, n$. In fact, if $K \cup f^{n-i}(L) \subset V_{i}$ and $0 \leq i<n$, since $K \cup f^{n-i}(L)$ and $C V_{i} \cap L$ are disjoint compact sets of $X$, there exists an open subset $W$ of $X$ such that

$$
K \cup f^{n-i}(L) \subset W \subset \bar{W} \subset V_{i} \cup C L .
$$

Define $V_{i+1}=f^{-1}(W)$; since $f(K) \cup f\left(f^{n-(i+1)}(L)\right) \subset K \cup f^{n-i}(L) \subset W$, we have $K \cup f^{n-(i+1)}(L) \subset V_{i+1}$; and $f\left(V_{i+1}\right) \subset \bar{W} \subset V_{i} \cup C L$ implies $L \cap \overline{f\left(V_{i+1}\right)} \subset V_{i}$. Beginning with $K \cup f^{n}(L) \subset K \subset V_{0}$, we define, by induction $V_{1}, \ldots, V_{n}$ with the desired properties.

Putting $U=V_{0} \cap V_{1} \cap \ldots \cap V_{n}$, we have $K^{\prime} \subset K \subset U$ and

$$
f(U) \subset f\left(V_{0}\right) \cap f\left(V_{1}\right) \cap \ldots \cap f\left(V_{n}\right) \subset L \cap \overline{f\left(V_{1}\right)} \cap \ldots \cap \overline{f\left(V_{n}\right)},
$$

hence

$$
\overline{f(U)} \subset\left(L \cap \overline { f ( V _ { 1 } ) } \cap \ldots \cap \left(L \cap \overline{f\left(V_{n}\right)} \cap L \subset V_{0} \cap \ldots V_{n-1} \cap V_{n}=U,\right.\right.
$$

but $\overline{f(U)}$ is compact since $\overline{f(U)} \subset L$. Moreover,

$$
X \subset \bigcup_{i=1}^{n} f^{-i}\left(K^{\prime}\right) \subset \bigcup_{i=1}^{\infty} f^{-i}(U) .
$$

Theorem 4.7. $f \in \mathbb{C} \mathbb{A}(X) \Rightarrow f \in \mathbb{C} \mathbb{C}(X)$.

Proof. Let $f: X \rightarrow X$ be a compact attraction map, $K$ a compact attractor for $f$ and $W$ an open set of $X$ such that $K \subset W$ and $L=\overline{f(W)}$ is compact. We have $L \subset X \subset \bigcup_{i=0}^{\infty} f^{-i}(W)$ hence, since $L$ is compact, there exists $n \in \mathbb{N}$ such that 
$L \subset \bigcup_{i=0}^{n} f^{-i}(W)$. Define $V=\bigcup_{i=0}^{n} f^{-i}(W)$; then

$$
\begin{gathered}
X \subset \bigcup_{i=0}^{\infty} f^{-i}(W) \subset \bigcup_{i=0}^{\infty} f^{-i}(V), \\
f(V) \subset \bigcup_{i=0}^{n} f^{-i+1}(W) \subset f(W) \cup V \subset L \cup V \subset V
\end{gathered}
$$

and

$$
f^{n+1}(V) \subset \bigcup_{i=0}^{n} f^{n-i+1}(W) \subset \bigcup_{j=0}^{n} f^{j+1}(W) \subset \bigcup_{j=0}^{n} f^{j}(L)
$$

which is compact and included in $V$, since $L \subset V$ and $f(V) \subset V$ implies that $f^{j}(L) \subset V$ for all $j \in \mathbb{N}$. Thus the contraction $\widetilde{f}: V \rightarrow V$ of $f$ is an eventually compact map. By 4.6, there exists an open set $U$ of $V$, hence of $X$, since $V$ is an open set, such that $\overline{\widetilde{f}(U)}=\overline{f(U)}$ is a compact of $U$ and $V \subset \bigcup_{n=0}^{\infty} \widetilde{f}^{-n}(U) \subset \bigcup_{n=0}^{\infty} f^{-n}(U)$; hence

$$
X \subset \bigcup_{i=0}^{\infty} f^{-i}(W) \subset \bigcup_{i=0}^{\infty} f^{-i}(V) \subset \bigcup_{i=0}^{\infty} f^{-i}(U)
$$

Theorem 4.8. $f \in \mathbb{A} \mathbb{S C}(X) \Rightarrow f \in \mathbb{C A}(X)$.

Pro of. Let $f: X \rightarrow X$ be an asymptotically compact map. Consider $\overline{C_{f}}$. It suffices to show that is a compact attractor for $f$. Then $f$ is a compact attraction map and 4.7 applies.

Let $V$ be an open subset of $X$ such that $\overline{C_{f}} \subset V$. Let $x \in X$. Put $K=\overline{\bigcup_{n=1}^{\infty} f^{n}(x)}$. Note that the family $\left\{\bigcap_{i=0}^{n} f^{i}(K) \cap C V\right\}_{n \in \mathbb{N}}$ of closed subsets of the compact $K$, has empty intersection, hence there exists a finite empty intersection. Therefore there exists $n \in \mathbb{N}$ such that $\bigcap_{i=0}^{n} f^{i}(K) \subset V$. Since

$$
f^{2 n}(x) \in \bigcup_{n=0}^{\infty} f^{n+i}(x)=f^{i}\left(\bigcup_{n=0}^{\infty} f^{n}(x)\right) \subset f^{i}(K)
$$

for every $0 \leq i \leq n$. Thus $f^{2 n}(x) \in \bigcap_{i=0}^{n} f_{i}(K) \subset V$ and $x \in f^{-2 n}(V)$.

We would like to emphasize that the Definitions 4.3, 4.4 and 4.5 are of geometrical origin. Definition 4.2 is abstract and introduced to make the proof of Schauder Fixed Point Theorem convenient to study.

The following diagram, as we proved earlier, shows the relations between these classes of functions:

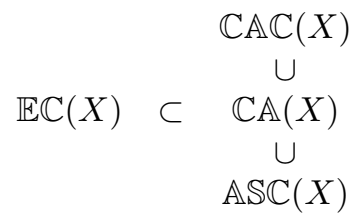


Moreover, if $f \in \mathbb{E} \mathbb{C}(X)$ satisfies an additional condition:

$$
\bigcup_{n=1}^{\infty}\left\{f^{n}(x)\right\} \text { is relatively compact for every } x \in X
$$

then $f \in \mathbb{A} \mathbb{S} \mathbb{C}(X)$.

Note that the fact

$$
\mathbb{K}(X) \subset \mathbb{E} \mathbb{C}(X) \cap \mathbb{A} \mathbb{S} \mathbb{C}(X)
$$

yields that the classes introduced in this section are not artificial.

However, the following important question still remains open:

Open PROBLEM. Are any of the inverse inclusions to those in the above diagram satisfied?

In what follows: for a given absorbing contraction map $f: X \rightarrow X$ we fix $U$ which satisfies conditions of 4.2. Then we let:

$$
\tilde{f}:(X, U) \rightarrow(X, U), \quad \text { where } \tilde{f}(x)=f(x) \text { for every } x \in X .
$$

Proposition 4.9. Let $f: X \rightarrow X$ be a compact absorbing contraction and $K$ be a compact subset of $X$. Then there exist $n \in \mathbb{N}$ such that $f^{n}(K) \subset U$.

Proof. From 4.2 we have for every $x \in X$ a number $n_{x} \in X$ such that $f^{n_{x}}(x) \in U$. Note that $\left(f^{n_{x}}\right)^{-1}(U)$ is an open set. Consider a covering $\left\{\left(f^{n_{x}}\right)^{-1}(U)\right\}_{x \in K}$. We choose a finite family of sets $\left\{\left(f^{n_{i}}\right)^{-1}(U)\right\}_{i=1}^{k}$. We end the proof putting $n=\max \left\{n_{1}, \ldots, n_{k}\right\}$.

As an easy consequence of the previous theorem and the fact of applying Cech homology functor $H$ with compact carriers and coefficients in the field of rational numbers $\mathbb{Q}$ we obtain:

THEOREM 4.10. Let $f$ be a compact absorbing contraction map and $\tilde{f}:(X, U) \rightarrow$ $(X, U)$. Then $\widetilde{f}_{*}$ is weakly nilpotent.

5. Main results. Now we are in position to state the Schauder Fixed Point Theorem (cf. $[\mathrm{S}],[\mathrm{DG}],[\mathrm{D}]$ ) in the most general form for single-valued mappings and metric spaces (for non-metric case see $[\mathrm{FB}]$ or $[\mathrm{W}]$ ).

Theorem 5.1. Let $X \in \mathrm{AR}$ and $f \in \mathbb{C} \mathbb{C}(X)$. Then $f$ has a fixed point.

Proof. According to 4.2 we choose $U \subset X$ to be an open subset of $X$ and satisfying all properties of 4.2 . Then $U \in \mathrm{ANR}$. We consider $\tilde{f}:(X, U) \rightarrow(X, U)$ as in 4.2 and $f_{1}: U \rightarrow U, f_{1}(x)=f(x)$ for every $x \in U$.

Then, in view of 4.2 and 2.3.6 we obtain that $\tilde{f}$ is a Lefschetz map and $\Lambda(\widetilde{f})=0$. Since $f_{1}: U \rightarrow U$ is a compact map, then from 3.1.5 we get that $f_{1}$ is a Lefschetz map. Consequently, from 3.4 we infer that $f$ is a Lefschetz map and $\Lambda(f)=\Lambda\left(f_{1}\right)$. So if $\Lambda(f) \neq 0$, then $\Lambda\left(f_{1}\right) \neq 0$. Now by applying once again Theorem 3.1.5 we have that $f_{1}$ has a fixed point and hence $f$ has a fixed point.

Remark 5.2. Observe that in fact we have proved the following so-called Lefschetz Fixed Point Theorem: 
If $X \in \mathrm{ANR}$ and $f \in \mathbb{C A C}(X)$ then $f$ is a Lefschetz map and $\Lambda(f) \neq 0$ implies that $f$ has a fixed point.

Now, as a special case of 5.1 we obtain:

Corollary 5.3. Let $X$ be an AR-space or, in particular, a convex subset of a normed space and let $f: X \rightarrow X$ be a continuous map.

(1) If $f \in \mathbb{K}(X)$, then $f$ has a fixed point.

(2) If $f \in \mathbb{E} \mathbb{C}(X)$, then $f$ has a fixed point.

(3) If $f \in \mathbb{C A}(X)$, then $f$ has a fixed point.

(4) If $f \in \mathbb{A S C}(X)$, then $f$ has a fixed point.

Now, we are going to state some open problems connected with 5.1. First, let us remark that in fact theorem 5.1 can be deduced from the Lefschetz Fixed Point Theorem, formulated in 5.1, by use of algebraic topology tools. On the other hand it is well-known that (comp. $[\mathrm{S}],[\mathrm{D}],[\mathrm{DG}]$ ) that Schauder Fixed Point Theorem for compact maps can be proved without using algebraic topology, i.e. in an elementary way. Hence we state:

Open problems 5.4. Assume that $X \in \mathrm{AR}$ or, in particular, $X$ is a convex subset of a normed space and $f: X \rightarrow X$ is a continuous map. Is it possible to prove in an elementary way i.e. without using algebraic topology any of the following formulations of the Schauder Fixed Point Theorem if:

(1) $f \in \mathbb{C A C}(X)$,

(2) $f \in \mathbb{E} \mathbb{C}(X)$,

(3) $f \in \mathbb{C A}(X)$

(4) $f \in \mathbb{A} \mathbb{S C}(X)$.

Yet, the authors do not know an answer for $X=\mathbb{R}^{2}$ and $f$ such that $f^{2}: \mathbb{R}^{2} \rightarrow \mathbb{R}^{2}$ is a compact map.

We would like to recall also the well-known asymptotic conjecture:

5.5 Asymptotic conjecture. It is possible to drop the assumption that $f$ is locally compact in at least one from the following cases:

(1) $f \in \mathbb{C A C}(X)$,

(2) $f \in \mathbb{E} \mathbb{C}(X)$,

(3) $f \in \mathbb{C} \mathbb{A}(X)$,

(4) $f \in \mathbb{A} \mathbb{S C}(X)$.

6. Admissible maps. This section is devoted to a large class of so-called admissible maps (see $[\mathrm{LG}],[\mathrm{FG}]$ for details) for which Schauder Fixed Point Theorem remains true.

We begin with the relevant definitions and facts.

We use letters $\phi, \varphi$ to denote multi-valued maps. The single valued maps are denoted by $f, g, p, q \ldots$ Let $\varphi: X \rightarrow Y$ be a multi-valued map. We associate with $\varphi$ the following diagram of continous maps:

$$
X \stackrel{p_{\varphi}}{\longleftarrow} \Gamma_{\varphi} \stackrel{q_{\varphi}}{\longrightarrow} Y
$$


in which

$$
\Gamma_{\varphi}=\{(x, y) \in X \times Y \mid y \in \varphi(x)\}
$$

is a graph of $\varphi$ and the natural projections $p_{\varphi}$ and $q_{\varphi}$ are given by:

$$
p_{\varphi}(x, y)=x \quad \text { and } \quad q_{\varphi}(x, y)=y .
$$

The image of a subset $B \subset X$ under $\varphi$ is

$$
\varphi(A)=\bigcup_{x \in A} \varphi(x)
$$

The counter-image of a subset $B \subset Y$ under $\varphi$ is

$$
\varphi^{-1}(B)=\{x \in X \mid \varphi(x) \subset B\} .
$$

A multi-valued map $\varphi: X \rightarrow Y$ is called upper semi-continous (u.s.c.) provided (i) $\varphi(x)$ is compact, for every $x \in X$, and (ii) for each open set $V \subset Y$, the counter-image $\varphi^{-1}(V)$ is an open subset of $X$.

Proposition 6.1. If $\varphi: X \rightarrow Y$ is an u.s.c. map and $A$ is a compact subset of $X$, then the image $\varphi(A)$ of $A$ under $\varphi$ is compact.

A multi-valued map $\varphi: X \rightarrow Y$, is called compact provided the image $\varphi(X)$ of $X$ under $\varphi$, is contained in some compact subset of $Y$. Let $\varphi: X \rightarrow Y$ be a multi-valued map, $A$ a subset of $X$ and $B$ a subset of $Y$. If $\varphi(A) \subset B$, then the contraction of $\varphi$ to the pair $(A, B)$ is the multi-valued map $\varphi^{\prime}: A \rightarrow B$ defined by $\varphi^{\prime}(a)=\varphi(a)$ for each $a \in A$. A contraction of $\varphi$ to the pair $(A, Y)$ is simply the restriction $\left.\varphi\right|_{A}$ of $\varphi$ to $A$. Let $\varphi: X \rightarrow X$ be a multi-valued map and let $A$ be a subset of $X$. If $\varphi(A) \subset A$, then $A$ is called an invariant subset under $\varphi$. A point $x \in X$ is called a fixed point of $\varphi$ if $x \in \varphi(x)$.

Let $\varphi: X \rightarrow Y$ and $\psi: Y \rightarrow Z$ be two multi-valued maps. Then the composition of $\varphi$ and $\psi$ is a multi-valued map $\psi \circ \varphi: X \rightarrow Z$ defined by $\psi \circ \varphi(x)=\psi(\varphi(x))$ for each $x \in X$. For a multi-valued map $\varphi: X \rightarrow X$ we denote by $\varphi^{m}, m \geq 1$, the $m$-th iteration of $\varphi$; by $\varphi^{0}$, we denote the identity map $\operatorname{Id}_{X}$.

Note that:

Proposition 6.2. The composition of u.s.c. maps is also u.s.c.

Lemma 6.3. Let $\varphi: X \rightarrow X$ be an u.s.c. map and let $U$ be an open invariant set under $\varphi$. Assume that, for every $x \in X$, there exists an integer $n$ such that $\varphi^{n}(x) \subset U$. Then, for every compact subset $K$ of $X$, there exists an integer $m$ such that $\varphi^{m}(K) \subset U$.

Obviously the Definitions $4.2-4.5$, can be rewritten for multi-valued maps.

An u.s.c. map $\varphi: X \rightarrow Z$ is said to be acyclic provided the set $\varphi(x)$ is acyclic for every point $x \in X$. We observe that if $\varphi: X \rightarrow Z$ is an acyclic map, then the natural projection $p_{\varphi}: \Gamma_{\varphi} \rightarrow Z$ is a Vietoris map.

Let $\varphi: X \rightarrow Z$ be a multi-valued map; a pair $(p, q)$ (of a single-valued, continuous) maps of the form $X \stackrel{p}{\stackrel{4}{\longleftarrow}} Y \stackrel{q}{\longrightarrow} Z$ is called a selected pair of $\varphi$ (we use notation $(p, q) \subset \varphi$ ), if the following conditions are satisfied:

(1) $p$ is a Vietoris map,

(2) $q\left(p^{-1}(x)\right) \subset \varphi(x)$ for each $x \in X$. 
Observe that, if $\varphi$ is a compact map and $(p, q) \subset \varphi$, the $q$ is also compact.

Definition 6.4. An u.s.c. map $\varphi$ is called admissible provided there exists a selected pair $(p, q)$ of $\varphi$.

Every acyclic map and, in particular, every continuous single-valued map is admissible; here the pair $\left(p_{\varphi}, q_{\varphi}\right)$ is a selected pair of $\varphi$. We note that the composition of admissible maps is also admissible.

Definition 6.5. An admissible map $\varphi: X \rightarrow X$ is called a Lefschetz map provided, for each selected pair $(p, q) \subset \varphi$, the linear map $q_{*} p_{*}^{-1}: H(X) \rightarrow H(X)$ is a Leray endomorphism.

If $\varphi: X \rightarrow X$ is a Lefschetz map, then we define the Lefschetz $\operatorname{set} \boldsymbol{\Lambda}(\varphi)$ of $\varphi$ by putting

$$
\boldsymbol{\Lambda}(\varphi)=\left\{\Lambda\left(q_{*} p_{*}^{-1}\right) \mid(p, q) \subset \varphi\right\}
$$

If $\varphi$ is an acyclic Lefschetz map, then the set $\boldsymbol{\Lambda}(\varphi)$ is a singleton which will be denoted by $\Lambda(\varphi)$.

Let $\varphi: X \rightarrow X$ be an u.s.c. map and $U$ be an open invariant subset of $X$ under $\varphi$. Assume that, for every $x \in X$, there exists an integer $n$ such that $\varphi^{n}(x) \subset U$. Let $(p, q)$ be a selected pair of $\varphi$ of the form $X \stackrel{p}{\longleftarrow} Y \stackrel{q}{\longrightarrow} Z$. Define $\widetilde{p}:\left(Y, p^{-1}(U)\right) \rightarrow(X, U)$, $\widetilde{q}:\left(Y, p^{-1}(U)\right) \rightarrow(X, U)$ by putting $\widetilde{p}(y)=p(y)$ and $\widetilde{q}(y)=q(y)$, for every $y \in Y$. Observe that $\widetilde{p}$ is a Vietoris map. Then, since $\mathrm{H}$ is a homology functor with compact carriers and $\left(q p^{-1}\right)^{n}(K) \subset \varphi^{n}(K)$, we have:

Lemma 6.6. The endomorphism $\widetilde{q}_{*} \widetilde{p}_{*}^{-1}: H(X, U) \rightarrow H(X, U)$ is weakly-nilpotent.

Let us observe, that the Theorem 3.1.5 can be generalized onto the case of multi-valued mappings as follows (comp. $[\mathrm{LG}]$ ):

TheOrEm 6.7. If $X \in \mathrm{ANR}$ and $\varphi: X \rightarrow X$ is an admissible map such that $q$ is compact for any $(p, q) \subset \varphi$. Then:

(i) $\varphi$ is a Lefschetz map,

(ii) $\boldsymbol{\Lambda}(\varphi) \neq\{0\}$ implies that $\varphi$ has a fixed point.

Remark 6.8. Note that if $X \in \mathrm{AR}$, then $\boldsymbol{\Lambda}(\varphi)=\{1\}$.

Now, we can define an admissible map $\varphi: X \rightarrow X$ to be:

(1) compact,

(2) locally compact,

(3) compact absorbing contraction,

(4) eventually compact,

(5) compact attraction,

(6) asymptotically compact,

analogously as (for single-valued maps) in Section 4 (comp. 4.1-4.5).

Then all results of Section 5 can be formulated for multi-valued maps and the same problems remain open (comp. 5.4). 


\section{References}

[B] K. Borsuk, Theory of Retracts, Monografie Matematyczne PAN, PWN Warszawa 1967.

[CB] C. Bowszyc, Some theorems in the Theory of Fixed Points, (Thesis), University of Warsaw (1969), (in Polish).

[FB] F. E. Browder, The fixed point theory of multi-valued mappings in topological vector spaces, Math. Ann. 177 (1968), 283-301.

[D] K. Deimling, Nonlinear Functional Analysis, Springer-Verlag Berlin Heidelberg New York Tokyo 1985.

[DG] J. Dugundji and A. Granas, Fixed Point Theory, Vol. 1, Monografie Matematyczne PAN, PWN Warszawa 1982.

[FG] G. Fournier and L. Górniew icz, The Lefschetz fixed point theorem for some noncompact multi-valued maps, Fundamenta Mathematicae XCIV (1977).

[F1] G. Fournier, Théorème de Lefschetz, I - Applications éventuellement compactes, Bull. Acad. Polon. Sci. 6 (1975), 693-701.

[F2] G. Fournier, Théorème de Lefschetz, II - Applications d'attraction compacte, ibid., 701-706.

[F3] G. Fournier, Théorème de Lefschetz, III - Applications asymptotiquement compactes, ibid., 707-713.

[LG] L. Górniewicz, Homological methods in fixed point theory of multivalued maps, Dissertationes Math. 129 (1976), Warszawa.

[AG] A. Granas, Points Fixes pour les Applications Compactes: Espaces de Lefschetz et la Theorie de l'Indice, SMS, Montreal 68 (1980).

[AG1] A. Granas, Generalizing the Hopf-Lefschetz fixed point theorem for non-compact ANR-s, Symposium on Infinite Dimensional Topology, Bâton-Rouge, 1967.

[S] J. Schauder, Der Fixpunktsatz in Funktionalräumen, Studia Math. 2 (1930), 171-180.

[W] A. Wieczorek, Survey of Results on Kakutani Property of Spaces with generalized Convexity, Fixed Point Theory and its Applications, Pitman Research Notes in Mathematics Series No. 252 (1990), 453-461. 